PROCEEDINGS OF THE

AMERICAN MATHEMATICAL SOCIETY

Volume 131, Number 2, Pages 337-341

S 0002-9939(02)06547-4

Article electronically published on May 22, 2002

\title{
THE INFLUENCE OF MINIMAL SUBGROUPS ON THE STRUCTURE OF A FINITE GROUP
}

\author{
YANGMING LI AND YANMING WANG
}

(Communicated by Stephen D. Smith)

\begin{abstract}
We study the detailed structure of a finite group under the assumption that all minimal subgroups of the generalized Fitting subgroup of some normal subgroup of $G$ are well-suited in $G$.
\end{abstract}

\section{INTRODUCTION}

All groups considered in this paper will be finite. We use conventional notions and notations, as in Huppert [3].

Recall that a minimal subgroup of a finite group is a subgroup of prime order. For groups of even order, it is helpful to also consider the cyclic subgroups of order 4. Two subgroups $H$ and $K$ of a group $G$ are said to permute if $H K=K H$. It is easily seen that $H$ and $K$ permute iff the set of $H K$ is a subgroup of $G$. We say, following Kegel [6], that a subgroup of $G$ is $\pi$-quasinormal in $G$ if it permutes with every Sylow subgroup of $G$. A number of authors have considered how minimal subgroups can be embedded in a group. Buckley [2] proved that if $G$ is a group of odd order and all minimal subgroups of $G$ are normal in $G$, then $G$ is supersolvable. Later Shaalan 7. proved that if $G$ is a finite group and every cyclic subgroup of prime order or of 4 is $\pi$-quasinormal in $G$, then $G$ is supersolvable. Some authors extend these results using formation theory $([10],[11)$. It is natural to limit the hypotheses of minimal subgroups to a smaller subgroup of $G$. Since every nonabelian simple group has a trivial Fitting subgroup, one cannot expect a detailed structure if one gives conditions on minimal subgroups of $F(G)$. So some authors have assumed the solvability of some normal subgroup of $G$ (i.e., the solvability of $G$ ) to get the structure of $G$, such as Asaad proved in [1: Let $\mathcal{F}$ be a saturated formation containing $\mathcal{U}$, the class of all supersolvable groups and suppose that $G$ is a group with a normal solvable subgroup $H$ such that $G / H \in \mathcal{F}$. If every subgroup of $F(H)$ of prime order or of 4 is $\pi$-quasinormal in $G$, then $G \in \mathcal{F}$. It is meaningful to remove the solvability of $H$ in the hypotheses of this result, but in this case, the Fitting subgroup $F(H)$ will sometimes be a trivial group in some instances as we mentioned above. However, as we show in this paper, we can obtain our results

Received by the editors July 30, 2001 and, in revised form, September 5, 2001.

2000 Mathematics Subject Classification. Primary 20D10, 20 D20.

Key words and phrases. $\pi$-quasinormal subgroup, supersolvable group, the generalized Fitting subgroup.

This project was supported by the Advanced Academic Center of ZSU and by a Grant of Education Ministry of China. 
about the structure of $G$ if we assume that the minimal subgroups of the generalized Fitting subgroup of some normal subgroups of $G$ are well suited in $G$.

Definition. Let $p$ be a prime and $G$ be a group. We define:

$$
\begin{aligned}
& \mathcal{P}_{p}(G)=\{x|x \in G,| x \mid=p\}, \\
& \mathcal{P}_{4}(G)=\{x|x \in G,| x \mid=4\}, \\
& \mathcal{P}(G)=\bigcup_{p \in \pi(G)} \mathcal{P}_{p}(G), \\
& \mathcal{P}^{*}(G)=\mathcal{P}_{4}(G) \cup \mathcal{P}(G) .
\end{aligned}
$$

An element $x$ of a group $G$ is said to be $\pi$-quasinormal in $G$ if $\langle x\rangle$ is $\pi$-quasinormal in $G$.

\section{Preliminaries}

Lemma 2.1 (6]). (1) A $\pi$-quasinormal subgroup of $G$ is subnormal in $G$. $K$.

(2) If $H \leq K \leq G$ and $H$ is $\pi$-quasinormal in $G$, then $H$ is $\pi$-quasinormal in

(3) If $H$ is a $\pi$-quasinormal Hall subgroup of $G$, then $H \triangleleft G$.

(4) Let $K \triangleleft G$ and $K \leq H$. Then $H$ is $\pi$-quasinormal in $G$ if and only if $H / K$ is $\pi$-quasinormal in $G / K$.

Lemma 2.2. If $P$ is a $\pi$-quasinormal p-subgroup of $G$ for some prime $p$, then $N_{G}(P) \geq O^{p}(G)$.

Proof. Now take $q$ any prime number distinct from $p$, and $Q$ any Sylow $q$-subgroup of $G$. Then $P Q$ is a subgroup of $G$. Now $P$ is a $\pi$-quasinormal, Sylow subgroup of $P Q$, so by Lemma 2.1(3), $P \triangleleft Q P$ and thus, $Q \subseteq N_{G}(P)$. Since $O^{p}(G)=\langle Q| Q \in$ $\left.\operatorname{Syl}_{q}(G), q \neq p\right\rangle$, thus $N_{G}(P) \geq O^{p}(G)$.

Let $G$ be a group. The generalized Fitting subgroup $F^{*}(G)$ of $G$ is the unique maximal normal quasinilpotent subgroup of $G$. Now $F^{*}(G)$ is an important subgroup of $G$ and is a natural generalization of $F(G)$. Its definition and important properties can be found in [5, X 13]. We would like to give the following basic facts which we will use in our proof.

Lemma 2.3. Let $G$ be a group and $M$ a subgroup of $G$. Then we have:

(1) If $M$ is normal in $G$, then $F^{*}(M) \leq F^{*}(G)$.

(2) $F^{*}(G) \neq 1$ if $G \neq 1$; in fact, $F^{*}(G) / F(G)=\operatorname{soc}\left(F(G) C_{G}(F(G)) / F(G)\right)$.

(3) $F^{*}\left(F^{*}(G)\right)=F^{*}(G) \geq F(G)$; if $F^{*}(G)$ is soluble, then $F^{*}(G)=F(G)$.

(4) Suppose $K$ is a subgroup of $G$ contained in $Z(G)$. Then $F^{*}(G / K)=$ $F^{*}(G) / K$.

Proof. (1)-(3) can be found in [5, X 13].

(4) Denote $F^{*}(G / K)=L / K$. Consider a chief series of $G$ of the form

$$
G=G_{0}>\cdots>G_{m-1}>G_{m}=K>G_{m+1}>\cdots>G_{n}=1 .
$$

By the definition of the generalized Fitting subgroup, $\forall x \in K, \bar{x}=x K$ induces an inner automorphism on the chief factor $\overline{G_{i-1}} / \overline{G_{i}}=\left(G_{i-1} / K\right) /\left(G_{i} / K\right)$ of $G / K$, for $i=1,2, \cdots, m$, thus $x$ induces an inner automorphism on the chief factor $G_{i-1} / G_{i}$ of $G$, for $i=1,2, \cdots, m$. Since $K \leq Z(G)$, the automorphism induced by $x$ on the chief factor $G_{i-1} / G_{i}$ of $G$ is identity, for $i=m+1, \cdots, n$, so $x$ induces an inner automorphism on the chief factor $G_{i-1} / G_{i}$ of $G$, for $i=1,2, \cdots, n$. Hence by [5] $\mathrm{X}$, Lemma 13.1], $x$ induces an inner automorphism on any chief factor of $G$. Thus $x \in F^{*}(G)$, i.e., $L \leq F^{*}(G)$. Obviously $F^{*}(G) \leq L$, hence $F^{*}(G / K)=F^{*}(G) / K$. 
Lemma 2.4. Suppose $G$ is a group with a normal subgroup $H$ such that $G / H$ is supersolvable. If every element of $\mathcal{P}^{*}(H)$ is $\pi$-quasinormal in $G$, then $G$ is supersolvable.

Proof. See [7, Theorem 3.1].

Lemma 2.5. Let $\mathcal{F}$ be a saturated formation containing $\mathcal{U}$ and let $G$ be a group. Then $G \in \mathcal{F}$ if and only if $G$ has a solvable normal subgroup $H$ such that $G / H \in \mathcal{F}$ and every element of $\mathcal{P}^{*}(F(H))$ is $\pi$-quasinormal in $G$. In particular, if $G$ is a group with a solvable normal subgroup $H$ such that $G / H$ is supersolvable and every element of $\mathcal{P}^{*}(F(H))$ is $\pi$-quasinormal in $G$, then $G$ is supersolvable.

Proof. See [1, Theorem and Corollary 1].

\section{RESUlts}

Theorem 3.1. Suppose $G$ is a group with a normal subgroup $N$ such that $G / N$ is supersolvable. If every element of $\mathcal{P}^{*}\left(F^{*}(N)\right)$ is $\pi$-quasinormal in $G$, then $G$ is supersolvable.

Proof. Suppose that the theorem is false and let $G$ be a counter-example of smallest order. Then we have:

(1) Every proper normal subgroup of $G$ is supersolvable.

If $H$ is a maximal normal subgroup of $G$, we have that $H / H \cap N$ is supersolvable, $F^{*}(H \cap N)$ is contained in $F^{*}(N)$ by Lemma 2.3. So $H, H \cap N$ satisfy the hypotheses of the theorem. The minimal choice of $G$ implies that $H$ is supersolvable.

(2) $N=G$, and $F^{*}(G)=F(G)<G$.

If $N<G$, then $N$ is supersolvable by (1), in particular, $N$ is solvable, so $F^{*}(N)=$ $F(N)$, then $G$ is supersolvable by applying Lemma 2.5 , a contradiction.

If $F^{*}(G)=G$, then $G$ is supersolvable by Lemma 2.4, a contradiction. Thus $F^{*}(G)<G$ is supersolvable. Since $F^{*}(G)$ is solvable, $F^{*}(G)=F(G)$ by Lemma $2.3(3)$.

(3) Contradiction.

(3.1) Now $G$ has a unique maximal normal subgroup, say $M$.

Suppose not; then there are maximal normal subgroups $M, N$. It follows that $G=N M$ and $G / M \cong N /(M \cap N)$ is supersolvable, as $N$ is. Now since $M$ is supersolvable, $G$ must be solvable. Hence, $G$ is supersolvable by Lemma 2.5, contrary to our assumption.

(3.2) $G / M$ is non-abelian simple and $G^{\prime}=G$.

By (3.1) $G / M$ is simple. If $G / M$ is cyclic group of prime order, then $G$ is solvable. This implies that $G$ is supersolvable by Lemma 2.5, which implies a contradiction. Thus $G / M$ is non-abelian simple; it follows that $G^{\prime} \not \leq M$, so $G^{\prime}=G$.

(3.3) $\forall q \in \pi(G), O^{q}(G)=G$.

If not, $O^{q}(G)<G$, then $O^{q}(G) \leq M$ is supersolvable. On the other hand, $G / O^{q}(G)$ is a $q$-group, so $G$ is solvable, a contradiction as in the proof of (3.2).

Since $F^{*}(G)=F(G)$ is not the identity group, we may choose a minimal prime divisor $q$ of $|F(G)|$, now that the Sylow $q$-subgroup $Q$ of $F(G)$ is a non-trivial normal subgroup of $G$. For every element $x$ of $\mathcal{P}^{*}(Q), x$ is $\pi$-quasinormal in $G$, thus $N_{G}(\langle x\rangle) \geq O^{q}(G)=G$ by Lemma 2.2. Thus $\langle x\rangle$ is normal in $G$. Since $G / C_{G}(\langle x\rangle)$ is abelian, we have that $G=G^{\prime} \leq C_{G}(\langle x\rangle)$, and therefore $G$ centralizes every element of $\mathcal{P}^{*}(Q)$. By Huppert's result ([4, Satz IV 5.12]), $G / C_{G}(Q)$ is a $q$-group. Since $G^{\prime}=G$, we have that $C_{G}(Q)=G$, i.e., $Q \leq Z(G)$. Hence by Lemma 2.3(4) 
$F^{*}(G / Q)=F^{*}(G) / Q$. Consider the group $\bar{G}=G / Q$. For any cyclic subgroup $\bar{L}$ of prime order $r$ of $F^{*}(\bar{G})$, since $Q$ is a Sylow $q$-subgroup of $F^{*}(G)$ with the minimal prime divisor, we have that $r>q$ (in particular, $r \neq 2$ ). Applying the Schur-Zassenhaus Theorem ([3]), we can denote $\bar{L}=L Q / Q$, where $L$ is a cyclic subgroup of order $r$ of $F^{*}(G)$. Since $L$ is $\pi$-quasinormal in $G$ by hypotheses, so $\bar{L}$ is $\pi$-quasinormal in $\bar{G}$. It follows that $\bar{G}$ satisfies the hypotheses of the theorem, and from the minimal choice of $G$ it follows that $G / Q$ is supersolvable and so $G$ is solvable, thus $G^{\prime}<G$, contrary to (3.2). The final contradiction. Thus, $G$ must be supersolvable, as required.

Denote the supersolvable residual of $G$ by $G^{\mathcal{U}}$. Using terminology of formations, it is easy to see that the above theorem can be expressed as the following:

Theorem 3.1'. Suppose $G$ is a group. Then $G$ is supersolvable if and only if every element of $\mathcal{P}^{*}\left(F^{*}\left(G^{\mathcal{U}}\right)\right)$ is $\pi$-quasinormal in $G$.

Corollary 3.2. Let $G$ be a group. If every element of prime order or order 4 of $F^{*}(G)$ is $\pi$-quasinormal in $G$, then $G$ is supersolvable.

Theorem 3.3. Let $\mathcal{F}$ be a saturated formation containing $\mathcal{U}$ and let $G$ be a group. Then $G \in \mathcal{F}$ if and only if $G$ has a normal subgroup $H$ such that $G / H \in \mathcal{F}$ and every element of $\mathcal{P}^{*}\left(F^{*}(H)\right)$ is $\pi$-quasinormal in $G$.

Proof. We only need to prove the "if " part.

By hypotheses every element of $\mathcal{P}^{*}\left(F^{*}(H)\right)$ is $\pi$-quasinormal in $G$, thus is $\pi$ quasinormal in $H$ by Lemma 2.1(2). Corollary 3.2 implies that $H$ is supersolvable, so $F^{*}(H)=F(H)$ and hence $G \in \mathcal{F}$ by applying Lemma 2.5 .

Using a proof similar to [1, Corollary 4], we also have a generalization of it.

Corollary 3.4. Let $\mathcal{F}$ be a saturated formation containing $\mathcal{U}$. Suppose that $G$ is a group with a normal subgroup $H$ such that $G / H \in \mathcal{F}$. Then $G \in \mathcal{F}$ under either of the following assumptions:

(a) $G$ is 2-nilpotent and every element of odd prime order of $F^{*}(H)$ is $\pi$ quasinormal in $G$.

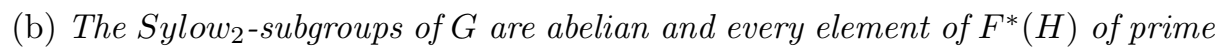
order is $\pi$-quasinormal in $G$.

Remark. Theorem 3.3 is not true for saturated formations which do not contain $\mathcal{U}$. For example, if $\mathcal{F}$ is the saturated formation of all nilpotent groups, then the symmetric group of degree three is a counterexample.

\section{ACKNOWLEDGMENT}

The authors thank the referee very much for his or her comments which simplified our proofs.

\section{REFERENCES}

1. M. Asaad and P. Csorgo. The influence of minimal subgroups on the structure of finite group. Arch. Math., 1999,72:401-404. MR 2000f:20022

2. J. Buckley. Finite groups whose minimal subgroups are normal. Math.Z, 1970,116:15-17. MR 41:6967

3. D. Gorenstein. Finite Groups. Chelsea (New York 1968). MR 38:229 MR 81b:20002

4. B. Huppert. Endliche Gruppen I. Berlin: Springer-Verlag, 1967. MR 37:302 
5. B. Huppert, N. Blackburn. Finite Groups III. New York, Berlin: Springer-Verlag, 1982. MR 84i:20001b

6. O. Kegel. Sylow-Gruppen und aubnormalteiler endlicher Gruppen. Math. Z., 1962, 78:205-221. MR 26:5042

7. A. Shaalan. The influence of $\pi$-quasinormality of some subgroups. Acta. Math. Hungar, 1990, 56:287-293. MR 92c:20034

8. Y. Wang. The influence of minimal subgroups on the structure of finite groups. Acta Math. Sinica, English series, 2000, 16:63-70. MR 2001c:20035

9. M. Weinstein (editor). Between nilpotent and solvable. Polygonal Publishing House, Passaic (1982). MR 84k:20002

10. A. Yokoyama. Finite soluble groups whose $\mathcal{F}$-hypercenter contains all minimal subgroups. Arch. Math., 1975,26:123-130. MR 53:3106

11. A. Yokoyama. Finite soluble groups whose $\mathcal{F}$-hypercenter contains all minimal subgroups II. Arch. Math., 1976,27:572-576. MR 56:5712

Department of Mathematics, Guangdong College of Education, Guangzhou, 510310, People's Republic of China

E-mail address: liyangming@gdei.edu.cn

Department of Mathematics, Zhongshan University, Guangzhou, 510275, People's RePUBLIC OF CHINA

E-mail address: stswym@zsu.edu.cn 